\title{
Ultra-sensitive RDT performance and antigen dynamics in a high-transmission Plasmodium falciparum setting in Mali
}

\author{
Emily N. Reichert ${ }^{1}$, Jen C. C. Hume², Issaka Sagara ${ }^{3}$, Sara A. Healy², Mahamadoun H. Assadou³, \\ Merepen A. Guindo ${ }^{3}$, Rebecca Barney ${ }^{1}$, Andy Rashid ${ }^{1}$, Ihn Kyung Yang ${ }^{1}$, Allison Golden ${ }^{1}$, Gonzalo J. Domingo ${ }^{1 *}$, \\ Patrick E. Duffy ${ }^{2}$ and Hannah C. Slater ${ }^{1^{*}}$ (1)
}

\begin{abstract}
Background: The recent expansion of tools designed to accurately quantify malaria parasite-produced antigens has enabled us to evaluate the performance of rapid diagnostic tests (RDTs) as a function of the antigens they detecttypically histidine rich protein 2 (HRP2) or lactate dehydrogenase (LDH).

Methods: For this analysis, whole blood specimens from a longitudinal study in Bancoumana, Mali were used to evaluate the performance of the ultra-sensitive HRP2-based Alere ${ }^{\mathrm{TM}}$ Malaria Ag P.f RDT (uRDT). The samples were collected as part of a transmission-blocking vaccine trial in a high transmission region for Plasmodium falciparum malaria. Furthermore, antigen dynamics after successful anti-malarial drug treatment were evaluated in these samples using the Q-Plex Human Malaria Array (4-Plex) to quantify antigen concentrations.

Results: The uRDT had a 50\% probability of a positive result at $207 \mathrm{pg} / \mathrm{mL}$ HRP2 [95\% credible interval (Crl) 160-268]. Individuals with symptomatic infection remained positive by uRDT for a median of 33 days [95\% confidence interval (CI) 28-47] post anti-malarial drug treatment. Biphasic exponential decay models accurately captured the population level post-treatment dynamics of both HRP2 and Plasmodium LDH (pLDH), with the latter decaying more rapidly. Motivated by these differences in rates of decay, a novel algorithm that used HRP2:pLDH ratios to predict if an individual had active versus recently cleared P. falciparum infection was developed. The algorithm had $77.5 \%$ accuracy in correctly classifying antigen-positive individuals as those with and without active infection.

Conclusions: These results characterize the performance of the ultra-sensitive RDT and demonstrate the potential for emerging antigen-quantifying technologies in the field of malaria diagnostics to be helpful tools in distinguishing between active versus recently cleared malaria infections.
\end{abstract}

Keywords: Malaria, Ultra-sensitive RDT, Antigenemia, HRP2, pLDH

\section{Background}

Malaria is most commonly diagnosed in humans by either microscopy or rapid diagnostic tests (RDTs), which detect antigens produced by malaria parasites $[1,2]$.

\footnotetext{
*Correspondence: gdomingo@path.org; hslater@path.org

${ }^{1}$ Diagnostics Program, PATH, Seattle, WA, USA

Full list of author information is available at the end of the article
}

The antigens histidine rich protein 2 (HRP2) and lactate dehydrogenase (LDH) are those most commonly targeted by RDTs. HRP2 antigen is expressed only by Plasmodium falciparum malaria, and RDTs targeting $P$. falciparum infections typically detect HRP2, while LDH is a constitutive enzyme expressed by all Plasmodium species. RDTs can specifically detect $P$. falciparum infections through antibodies targeting subspecies epitopes on the $\mathrm{LDH}$

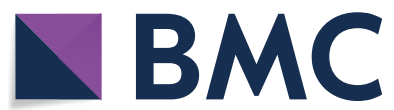

(c) The Author(s) 2020. This article is licensed under a Creative Commons Attribution 4.0 International License, which permits use, sharing, adaptation, distribution and reproduction in any medium or format, as long as you give appropriate credit to the original author(s) and the source, provide a link to the Creative Commons licence, and indicate if changes were made. The images or other third party material in this article are included in the article's Creative Commons licence, unless indicated otherwise in a credit line to the material. If material is not included in the article's Creative Commons licence and your intended use is not permitted by statutory regulation or exceeds the permitted use, you will need to obtain permission directly from the copyright holder. To view a copy of this licence, visit http://creativeco mmons.org/licenses/by/4.0/. The Creative Commons Public Domain Dedication waiver (http://creativecommons.org/publicdomain/ zero/1.0/) applies to the data made available in this article, unless otherwise stated in a credit line to the data. 
antigen, as well as all Plasmodium species through conserved epitopes. Of note, HRP2-based RDTs can exhibit cross-reactivity with HRP3 due to antigenic similarity.

Until recently, malaria RDT evaluation programmes have focused on benchmarking malaria diagnostic tests against parasite density measured either by microscopy or nucleic acid tests, although antigen concentrations of standard samples have recently begun to be included [35]. RDTs have been considered to perform equivalently to microscopy in terms of sensitivity and specificity [6], with a recognition that HRP2-based RDTs may provide false positives due to the long half-life of circulating HRP2 [1, 2]. The advent of new RDTs, such as the ultra-sensitive HRP2-based Alere ${ }^{\text {TM }}$ Malaria Ag P.f RDT (Abbott, South Korea) with a tenfold lower limit of detection for HRP2 than previous RDTs $[7,8]$ has incentivized the research community to better understand antigen dynamics in infected populations [8-12]. Concurrently, new assays for antigen quantification have been developed both as research tools on the Luminex platform [13, 14] and as the commercially available Q-Plex ${ }^{\mathrm{TM}}$ Human Malaria Array (Quansys Biosciences, USA) [9].

This report describes the performance of the ultrasensitive Alere Malaria Ag P.f RDT (uRDT) on samples collected in a phase 1 clinical trial malaria vaccine study in Bancoumana, Mali. This trial assessed Pfs25M-EPA/ Alhydrogel $^{\circledR}$ and Pfs230-EPA/Alhydrogel ${ }^{\circledR}$ individually and in combination for safety and functional immunogenicity in malaria-exposed Malian adults (ClinicalTrials.gov: NCT02334462). The vaccine was given on a 0 , 1, 6 month schedule in the 1st year with a booster dose 12 months after dose 3. Given the longitudinal nature of the study cohort, the population level antigen dynamics for both HRP2 and LDH were investigated, as well as relative antigen abundance post-treatment, as determined with the Q-Plex enzyme-linked immunosorbent assay (ELISA).

\section{Methods}

\section{Study design and data collection}

Individuals in this analysis were part of a double-blind, randomized, phase 1 clinical trial conducted by the Laboratory of Malaria Immunology and Vaccinology (LMIV)/National Institute of Allergy and Infectious Diseases (NIAID)/National Institutes of Health (NIH). Bancoumana is a rural village $60 \mathrm{~km}$ from Bamako, Mali, with high prevalence of Plasmodium falciparum. This trial investigated the safety and immunogenicity of Pfs230D1M-EPA/Alhydrogel ${ }^{\circledR}$ and Pfs25M-EPA/Alhydrogel, both transmission-blocking vaccines against $P$. falciparum. Transmission-blocking vaccines were administered on study days 0, 28, 169, and 540. Blood smears were prepared before each vaccination, at least monthly post vaccination, or when clinically indicated. Starting 1 week after the third and fourth vaccinations, blood smears were prepared twice a week for 6 weeks at the same time that subjects underwent direct skin feeding assays with colony-raised Anopheles coluzzii to assess malaria parasite transmission. Whole blood samples to be analysed in this study were collected at per-protocol scheduled blood draws 1-6 weeks after the fourth vaccination (study days 547, 554, 568, and 582) during peak malaria transmission season (July-December). Between 1 and $21 \mathrm{~mL}$ of whole blood was collected at each blood draw for each study participant, all who agreed to have blood samples stored for future research prior to enrollment. Individuals who at any point presented with symptomatic malaria, defined as any parasitaemia by blood smear or RDT positive result with symptoms consistent with malaria, were treated with anti-malarial drugs artemether-lumefantrine (Coartem or Laritem) for uncomplicated malaria and artemether for severe malaria. Whole blood samples for individuals treated with anti-malarials were collected at the same per-protocol frequency as the remainder of the study cohort. Microscopy-positive asymptomatic individuals were not treated, per Malian National Policy on Malaria Control Guidelines.

\section{Sample evaluation}

Frozen whole blood samples $(n=622)$ were sent to PATH's laboratory (Seattle, WA, USA) for further evaluation. Two ultra-sensitive HRP2-based Alere Malaria Ag P.f RDTs (uRDT), product number 05FK140, lots 05LDB005A and 05LDB004A, were used to test in duplicate each specimen, all of which had been stored at $-80{ }^{\circ} \mathrm{C}$. The test required $5 \mu \mathrm{L}$ of whole blood and was run following the standard workflow outlined in Das et al. [15]. A final uRDT result was generated from duplicate uRDT results in agreement only; when results were either discordant or invalid, results were considered not confirmed and excluded from final analyses.

HRP2 and Plasmodium LDH (pLDH) concentrations were quantified using the Q-Plex Human Malaria Array (4-Plex), which quantifies pLDH by detecting pan epitope [9]. Standards of recombinant protein with known antigen concentration are run on each plate allowing quantification through standard curves. Ranges of quantification for HRP2 and pLDH were $1.07-16,500 \mathrm{pg} / \mathrm{mL}$ and $14.41-525,700 \mathrm{pg} / \mathrm{mL}$, respectively. For numeric analyses, samples with antigen concentrations beyond the limit of quantification (LOQ) for Q-Plex were treated as (upper LOQ) * 2 and (lower LOQ)/2. Thresholds above which samples were defined as antigen positive, determined through receiver operating characteristics analysis to identify the optimal sensitivity and specificity tradeoff, 
were $2.30 \mathrm{pg} / \mathrm{mL}$ for HRP2 and $47.8 \mathrm{pg} / \mathrm{mL}$ for $\mathrm{pLDH}$ [9]. Parasite count by microscopy included both gametocytes and asexual parasites and was estimated as parasites per 1000 white blood cells (WBCs), but is reported in parasites $/ \mu \mathrm{L}$, using the conversion of $8000 \mathrm{WBCs} / \mu \mathrm{L}[16]$. Gametocyte counts were combined with asexual parasite counts as both have been shown to express HRP2 and LDH [17].

\section{Statistical analysis}

Data compilation and statistical analysis was performed using R 3.6.0 software [18]. A Bayesian logistic regression model with study level random effects was used to model the relationship between HRP2 concentration and probability of detection by uRDT. A $\log 10$ transformation was applied to the HRP2 concentration data and a Gaussian distribution with a mean of zero and standard deviation of three was used for the prior. Four chains of 1000 iterations were ran after a burn-in of 500 iterations, from which median predictions and 95\% Bayesian credible intervals (CrI) were taken. A Kaplan-Meier survival curve was generated to estimate probability of a uRDTpositive result for individuals post successful anti-malarial treatment. HRP2 and pLDH dynamics post-treatment were modelled by fitting monophasic and biphasic exponential decay models. A monophasic decay assumes a constant decay rate over time, whereas the biphasic decay model allows for two different decay rates, typically a rapid initial decay followed by a period of slower decay. The functional forms for these two models are:

$$
\begin{aligned}
& \text { Monophasic: } \log _{10} \text { (concentration) }=k_{1} t+C_{0} \\
& \text { Biphasic: } \log _{10} \text { (concentration) } \\
& \qquad=\left\{\begin{array}{lll}
k_{1} t & \text { if } & t<t_{\text {switch }} \\
k_{1} t+k_{2}\left(t-t_{\text {switch }}\right) & \text { if } & t \geq t_{\text {switch }}
\end{array}\right\}+C_{0}
\end{aligned}
$$

where $t=$ time (in days), $k_{1}, k_{2}$ are decay parameters, $t_{\text {switch }}$ is the switch point between "fast" and "slow" decay, and $\mathrm{C}_{0}$ is the $\log 10$ initial concentration. An individual level random effect was incorporated into each model, accounting for individual variation in antigen concentration at time of treatment $(t=0)$ and therefore fitting unique values of $C_{0}$ to each individual. Models were compared using ANOVA and those that minimized both Akaike information criterion (AIC) and Bayesian information criterion (BIC) were ultimately selected. Predictive intervals were obtained by using the predictInterval function in the R package merTools, which estimates the distribution of all model parameters while incorporating uncertainty in both fixed and random effects. This function was run over 1000 simulations to obtain $95 \%$ predictive intervals. Finally, receiver operating characteristic (ROC) curves were calculated to determine optimal thresholds for predicting active versus recently cleared P. falciparum infection, with thresholds maximizing Youden's index (the sum of sensitivity and specificity) defined as optimal.

\section{Results \\ Study population}

Blood samples were collected from 160 adults between the ages of 18-53 years living in Bancoumana or the surrounding area. Collections of interest were performed between September-November 2017, totaling 622 blood specimens. Mean participant age was 40.7 years [standard deviation (SD) 8.4] at time of blood draw visits. Out of 160 participants, 110 [69\%; 95\% confidence interval (CI); 61-76] were positive by ultra-sensitive rapid diagnostic test (uRDT) and 99 (62\%; 54\%-69\%) were positive by microscopy at least once during the sampling window. Furthermore, 101 (63\%; 55\%-71\%) of these participants presented with symptomatic infection at least once. Of the 166 microscopy-positive whole blood specimens, 155 (93\%) were $P$. falciparum only, 3 (1.8\%) were $P$. falciparum mixed infections, and $8(4.8 \%)$ were non- $P$. falciparum (Plasmodium ovale or Plasmodium malariae). Non-P. falciparum infections as identified by microscopy were excluded, leaving 614/622 (98.7\%) of specimens for further evaluation.

\section{Performance of the ultra-sensitive RDT}

The performance of the uRDT was evaluated against microscopy as well as HRP2 and all-malaria pLDH confirmed by Q-Plex (Table 1). There were ten samples (1.6\%) with discordant uRDT results excluded from uRDT evaluation results [geometric mean

Table 1 Performance of the uRDT against Q-Plex ELISA and microscopy detection

\begin{tabular}{llll}
\hline & $\begin{array}{l}\text { HRP2 Q-Plex ELISA } \\
\text { reference, \% (95\% } \\
\text { Cl) }\end{array}$ & $\begin{array}{l}\text { pLDH Q-Plex ELISA } \\
\text { reference, \% (95\% } \\
\text { Cl) }\end{array}$ & $\begin{array}{l}\text { Microscopy } \\
\text { reference, \% } \\
\mathbf{( 9 5 \% ~ C l ) ~}\end{array}$ \\
\hline Sensitivity & $58 \%(53-63)$ & $68 \%(63-74)$ & $78 \%(71-84)$ \\
Specificity & $100 \%(100-100)$ & $84 \%(80-88)$ & $74 \%(70-78)$ \\
PPV & $100 \%(100-100)$ & $78 \%(73-83)$ & $51 \%(45-57)$ \\
NPV & $53 \%(48-58)$ & $77 \%(72-81)$ & $90 \%(87-93)$ \\
\hline
\end{tabular}

Results are given with associated $95 \% \mathrm{Cls}$. Whole blood samples were defined as HRP2- and pLDH-positive with concentrations $>2.30 \mathrm{pg} / \mathrm{mL}$ and $>47.8 \mathrm{pg} /$ $\mathrm{mL}$, respectively. Microscopy-positive samples were those with any $P$. falciparum parasites detected per 1000 WBCs

ELISA enzyme-linked immunosorbent assay, LDH lactate dehydrogenase, PPV positive predicted value, NPV negative predicted value 
(GM): $190 \mathrm{pg} / \mathrm{mL}$ HRP2]. All uRDT-positive results were also HRP2-positive. Only 51\% of uRDT-positive infections were microscopy positive, whereas $73 \%$ of microscopy-positive infections were positive by uRDT (Fig. 1). The uRDT detected $80.7 \%$ of infections that were both HRP2 and microscopy-positive versus 56.1\%

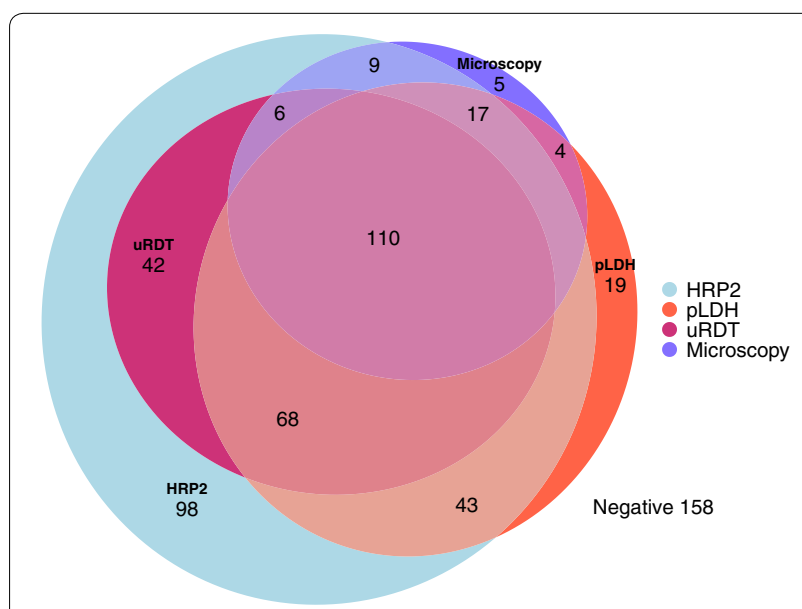

Fig. 1 Relationship between various methods of detection. Venn diagram visualizing overlap in detection between microscopy and new generation diagnostics (URDT and Q-Plex ELISA) for the 579/614 blood samples with conclusive results for all four detection methods. HRP2, histidine rich protein 2; pLDH, Plasmodium lactate dehydrogenase; URDT, ultra-sensitive rapid diagnostic test
HRP2-positive only, indicating a higher sensitivity performance for high-density infections. There were five microscopy-confirmed infections that were not confirmed by either HRP2, pLDH, or the uRDT, all of which had low parasitaemia ( $<16$ parasites $/ \mu \mathrm{L})$. Only two-thirds (66.2\%) of pLDH-positive samples also had sufficient HRP2 to be detected by uRDT.

Comparable uRDT positivity and HRP2 concentration data exists for two recently published studies conducted in asymptomatic individuals, one in Uganda $(n=607$, high transmission) [7], and one in Myanmar ( $\mathrm{n}=1847$, low transmission) [8]. Details of these studies have been previously published and are briefly summarized in Additional file 1 . Figure $2 \mathrm{a}$ shows the probability of detection by uRDT in relation to HRP2 concentration for Mali in comparison to the studies conducted in Uganda and Myanmar [7, 8]. Differences emerged in uRDT detection limits among the three study populations: there was a $50 \%$ probability of testing positive by uRDT at HRP2 thresholds of $207 \mathrm{pg} / \mathrm{mL}$ [95\% credible interval (CrI) 160-268] in Mali, $15 \mathrm{pg} / \mathrm{mL}$ (11-21) in Uganda, and $101 \mathrm{pg} / \mathrm{mL}(66-156)$ in Myanmar. The established limit of detection (LOD) for the commercial Alere Malaria Ag P.f uRDT is $80-100 \mathrm{pg} / \mathrm{mL}$, per laboratory evaluation by Das et al. [15]. The HRP2 distributions for samples in each study population stratified by uRDT result are visualized in Fig. 2b.
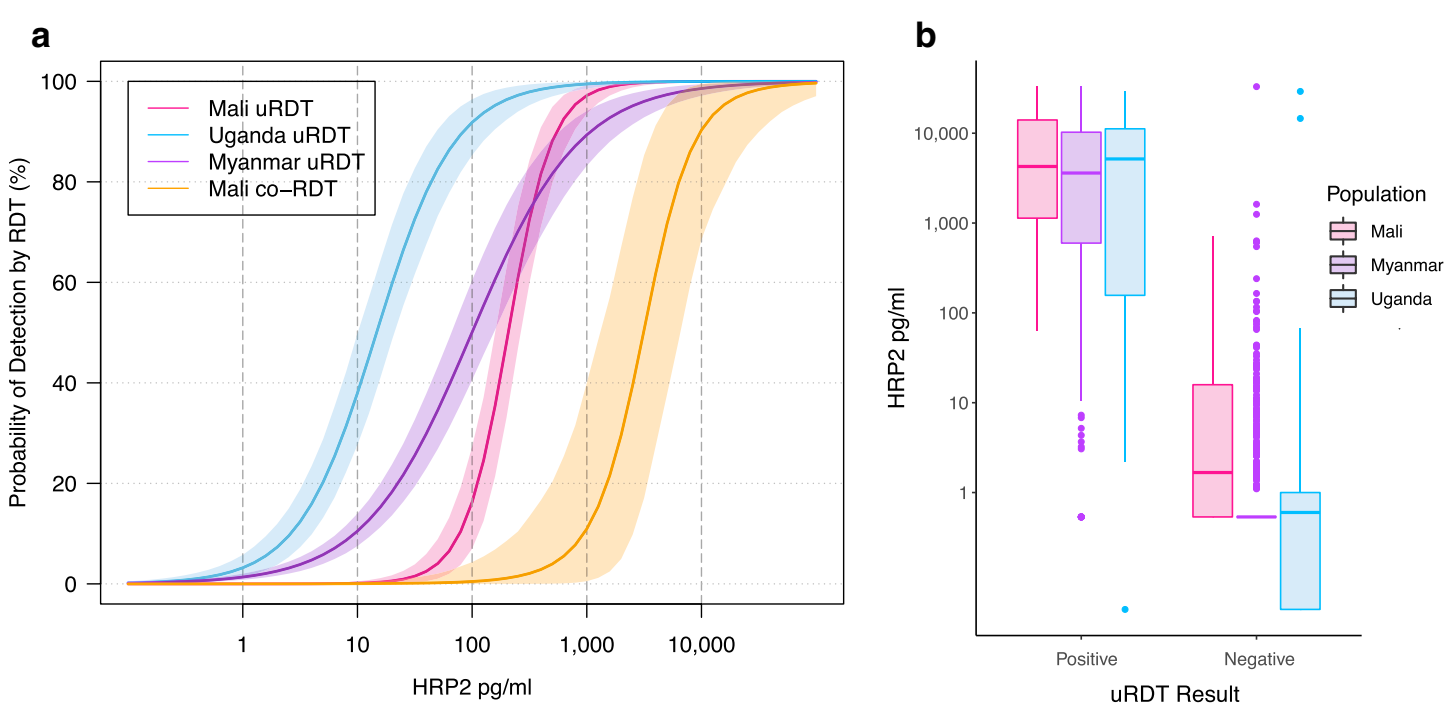

Fig. 2 Estimated probability of uRDT detection by HRP2 concentration. a Fitted relationship between uRDT performance (probability of detection) and HRP2 concentration for Mali $(n=614$, high transmission) and two other sample sites: Uganda ( $n=607$, high transmission) and Myanmar ( $n=1847$, low transmission) using a Bayesian logistic regression model. Probability of detection is also shown for a small number $(n=35)$ of specimens with a co-RDT run in the field in Mali (orange). Shaded regions show 95\% credible intervals for median model predictions. b Boxplot of HRP2 distributions in Mali, Myanmar, and Uganda study cohorts stratified by URDT result. Co-RDT conventional RDT, HRP2 histidine rich protein 2 , URDT ultra-sensitive rapid diagnostic test 
Compared to a small subset of samples in Mali for which conventional RDTs (co-RDTs) were run in the field $(n=35)$, the uRDT was on average more than tenfold more sensitive in its LOD than the co-RDT: $50 \%$ probability of detection was achieved at $207 \mathrm{pg} / \mathrm{mL}$ HRP2 for the uRDT compared to $3140 \mathrm{pg} / \mathrm{mL}$ HRP2 for co-RDT. However, both tests had higher detection thresholds than expected $(80-100 \mathrm{pg} / \mathrm{mL}$ for $\mathrm{uRDT}$ and $800 \mathrm{pg} / \mathrm{mL}$ for co-RDT) [15]. Out of 415 HRP2-positive samples, 104 (25\%) were between the calculated $50 \%$ detection threshold of the uRDT and co-RDT, with 37/104 (36\%) of these also microscopy positive for $P$. falciparum.

\section{Antigenaemia, detection, and treatment status}

In Mali, the geometric mean for HRP2 was $55.4 \mathrm{pg} / \mathrm{mL}$ (geometric SD 49.7) and for $\mathrm{pLDH}$ was $101.8 \mathrm{pg} / \mathrm{mL}$ (geometric SD 17.7). The correlation for the transformed $\log 10$ value of each antigen (pLDH and HRP2) with $\log 10$ parasitaemia by microscopy was poor, but was higher for
pLDH than HRP2 $\left(R^{2}=0.61\right.$ and $R^{2}=0.29$, respectively $)$ among all microscopy-positive samples (Additional file 1 : Figures S1 and S2).

Figure 3 shows the HRP2 and pLDH concentrations for each sample in the Mali study classified by both uRDT and microscopy result. Geometric mean (GM) HRP2 concentration was on average $4002 \mathrm{pg} / \mathrm{mL}$ for uRDTpositive samples and $3.2 \mathrm{pg} / \mathrm{mL}$ for uRDT-negative samples. Microscopy-positive samples also had higher concentrations of HRP2 (GM: $1430 \mathrm{pg} / \mathrm{mL}$, compared to $18 \mathrm{pg} / \mathrm{mL}$ for those microscopy-negative). Of interest, a majority $(63 \%)$ of microscopy-positive samples had $<10,000 \mathrm{pg} / \mathrm{mL} \mathrm{pLDH}$, below the estimated LOD for currently available pLDH-based diagnostic tests. Of those with $\mathrm{pLDH}>10,000 \mathrm{pg} / \mathrm{mL}$, only one sample had HRP $2<100 \mathrm{pg} / \mathrm{mL}$. A combined HRP2, pLDH diagnostic without improved sensitivity for pLDH would, therefore, not have captured a significantly higher number of

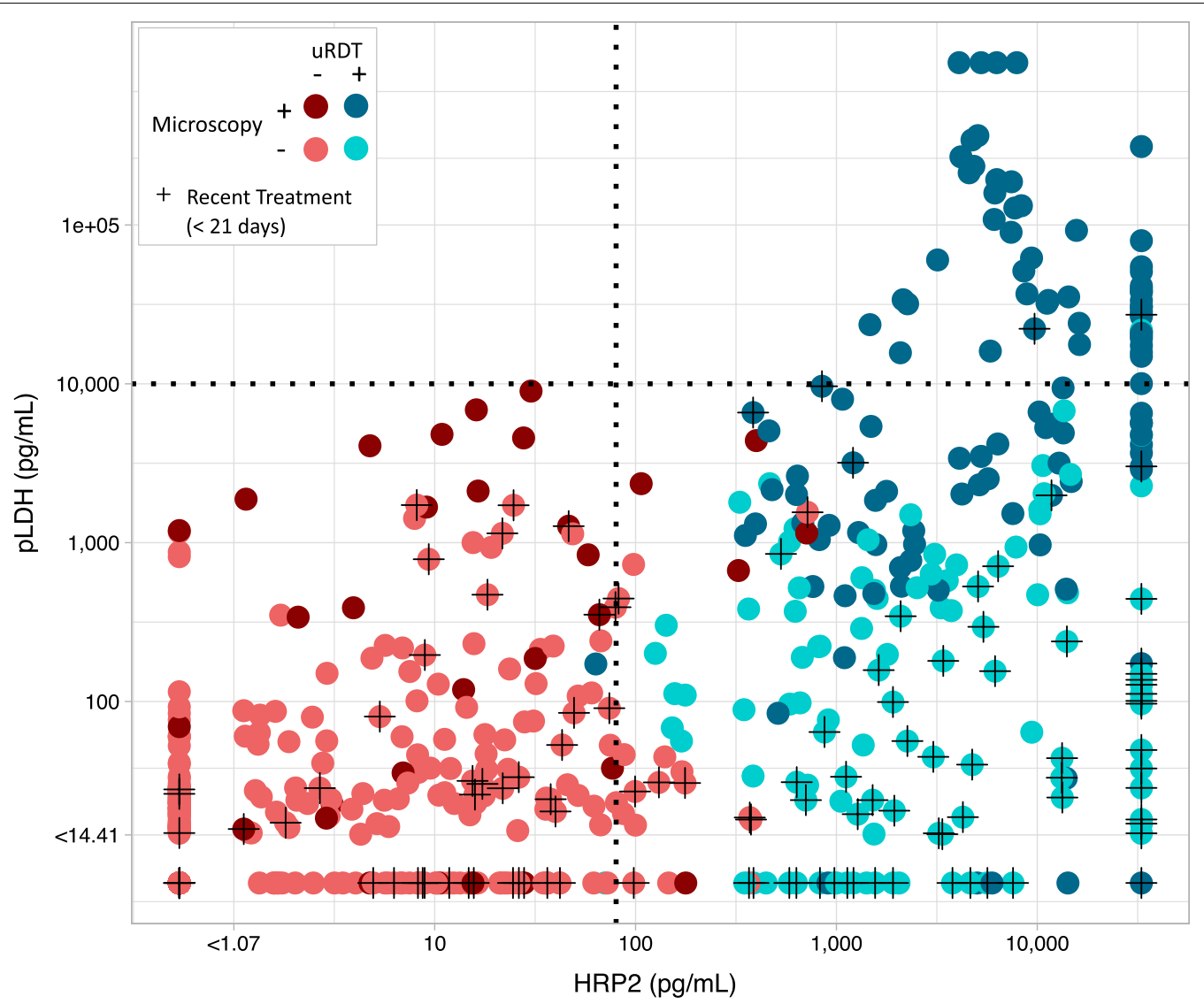

Fig. 3 Classification of diagnostic performance by relative biomarker concentrations. Each blood sample with results for microscopy, Q-plex, and URDT $(n=579)$ is represented as a single point colored by URDT and microscopy results. Dotted lines indicate the most sensitive thresholds for currently available RDTs: 10,000 pg/mL for pLDH, an estimate based on detection of $\sim 200$ parasites/ $/ \mathrm{LL}$ [9, 30, 31], and 80-100 pg/mL for HRP2 [15]. Additionally, black crosses indicate those with recent antimalarial drug treatment (previous 21 days). HRP2, histidine rich protein 2; pLDH, Plasmodium lactate dehydrogenase; RDT, rapid diagnostic test; uRDT, ultra-sensitive rapid diagnostic test 
infected individuals than the HRP2-based test alone in this high transmission P. falciparum setting.

Individuals who at any point in this study presented with symptomatic malaria were treated with anti-malarial drugs. There were 130 symptomatic infections treated in 101 unique individuals. Of samples from "recently treated" individuals, defined as receiving anti-malarial drugs in the previous 21 days, a majority (52.5\%) were still positive by uRDT, with the highest proportion (43.3\%) uRDT-positive and microscopy-negative (Fig. 3). Recently treated samples have considerably lower values of $\mathrm{pLDH}$ (GM: $50 \mathrm{pg} / \mathrm{mL}$ ) compared to samples from individuals that have not recently been treated (GM: $156 \mathrm{pg} / \mathrm{mL}$ ). The opposite is true for HRP2 concentration $(367 \mathrm{pg} / \mathrm{mL}$ compared to $91 \mathrm{pg} / \mathrm{mL}$ ). A Kaplan-Meier survival curve fitted to samples from individuals up to 70 days post-treatment $(\mathrm{n}=187)$ with no evidence of persistent $P$. falciparum infection by microscopy (i.e. no microscopy-positive result at any timepoint after 1 day post-treatment) estimated that median time to uRDT negativity was 33 days post-treatment (95\% CI 28-47) (Fig. 4).

\section{Antigen dynamics post-treatment}

Monophasic and biphasic exponential decay models with individual-level random effect were fitted to HRP2 and pLDH data for samples with no microscopic evidence of recurrent $P$. falciparum post-treatment. Models were fit up to 35 days post-treatment for HRP2 (152 samples, 75 individuals) and only 8 days for pLDH (39 samples, 37 individuals) to avoid uncertainty in model predictions once median antigen values declined below the limit of quantification. Using criterion that minimized both AIC and BIC, a biphasic exponential model best estimated population level antigen decay (Fig. 5) and was a significantly better fit compared to monophasic for both HRP2 and $\mathrm{pLDH}(\mathrm{P}=0.02$ and $\mathrm{P}=0.003$, respectively). The optimal switch point (knot) was 2 days for pLDH and 3 days for HRP2. Decay parameters for $\mathrm{pLDH}$ were $\mathrm{k}_{1}=-1.83$ and $\mathrm{k}_{2}=1.80$ compared to $\mathrm{k}_{1}=-0.57$ and $\mathrm{k}_{2}=0.53$ for HRP2; pLDH initially decayed more rapidly than HRP2, as is consistent with the literature [19]. Based on median model predictions, the average time to reach "undetectable" levels of $<100 \mathrm{pg} / \mathrm{mL}$ was 3 days for pLDH compared to 26 days for HRP2 among this study cohort. It is important to note that fitted median antigenaemia at time of treatment was fairly low compared to other studies $(380,000 \mathrm{pg} / \mathrm{mL} \mathrm{pLDH}$ and $28,000 \mathrm{pg} / \mathrm{mL}$ HRP2) [19], potentially because participants were being treated quickly as soon as symptomatic infection presented due to frequent study visits.

Differing initial rates of decay between HRP2 and pLDH post-treatment indicated the ratio of these

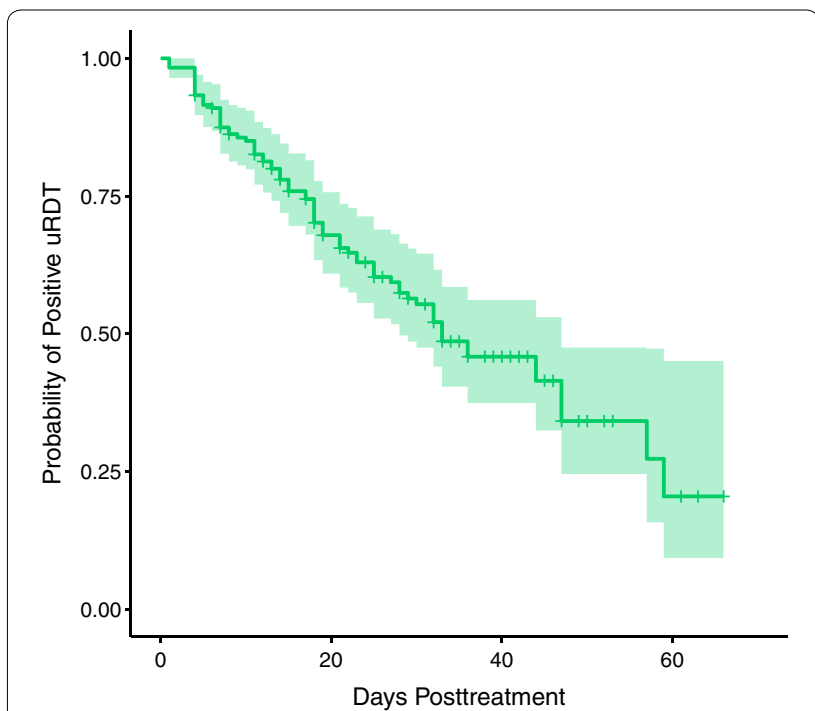

Fig. 4 Persistence in uRDT detection post-treatment. A KaplanMeier survival curve showing the probability of remaining URDT positive following successful treatment for symptomatic $P$. falciparum infection. The shaded region represents the median model's $95 \% \mathrm{Cl}$. Median time to URDT negativity was 33 days ( $95 \% \mathrm{Cl} 28-47)$, with day of antimalarial drug treatment for symptomatic infection defined as day 0. Samples with evidence of unsuccessful treatment or recurrent infection, defined as those positive for P. falciparum by microscopy at any point after 1 day post-treatment, were excluded. URDT ultra-sensitive rapid diagnostic test

antigens' concentrations may differ for recently treated individuals. Using only samples within 4 weeks pretreatment to 7 weeks post-treatment (311 samples from 89 individuals), HRP2:pLDH ratios were calculated. Samples from six study participants treated twice during this time frame were included as separate samples with differing days of treatment. The median HRP2:pLDH ratio was 0.348 [interquartile range (IQR): $0.07-2.11$ ] pretreatment and 3.41 (IQR: 0.28-51.0) post-treatment (Fig. 6a). HRP2:pLDH ratios were significantly elevated at weeks 1-3 post-treatment, so "recent treatment" was, therefore, defined as treatment with anti-malarial drugs within the past 21 days. Motivated by this difference, attempts were made to distinguish recently cleared but antigen-positive $P$. falciparum infection from active infection based on the HRP2:pLDH ratio. Active infections were defined as those positive for $P$. falciparum by microscopy, whereas cleared infections were defined as microscopy-negative but recently treated $(<21$ days ago) with persistent antigenaemia (HRP2 and/or PLDH positive). The relationship between the ratio, HRP2 concentration, and recent treatment history is visualised in (Fig. 6b). Recently treated individuals with successful clearance of $P$. falciparum parasitaemia appeared clustered above different threshold ratios over and under $100 \mathrm{pg} / \mathrm{mL}$ HRP2, as confirmed 

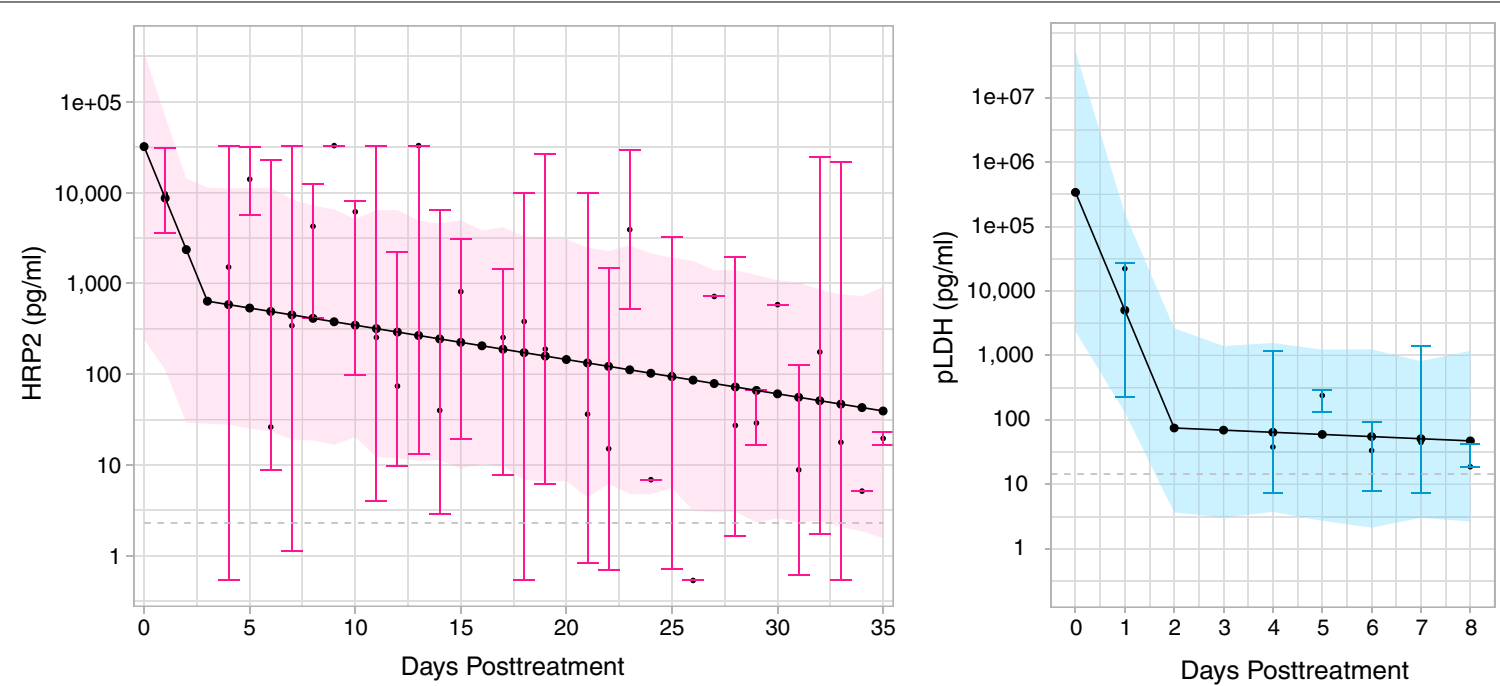

Fig. 5 Biphasic exponential decay models for HRP2 and pLDH post-treatment. Fitted population-level biphasic decay models, with a switch time (knot) of 3 days post-treatment for HRP2 (pink) and 2 days post-treatment for pLDH (blue). Black dots indicate median model predictions, with shaded regions showing associated 95\% prediction intervals. Median antigen concentrations with 95\% Cls, depicted by pink and blue vertical lines, are also plotted for each day data were available post-treatment. Horizontal grey lines indicate the lower LOQ for each antigen-values below the $L O Q$ were set to half this $L O Q$ and retained in the analyses. Samples with evidence of recurrent $P$. falciparum parasitemia by microscopy at any point in the follow-up window after 1 day post-treatment were excluded. HRP2, histidine rich protein 2; pLDH, Plasmodium lactate dehydrogenase

by ROC curve analysis. A pair of values (for HRP2 and HRP2:pLDH) were then selected such that sensitivity and specificity were maximized. If HRP2 is $>100 \mathrm{pg} / \mathrm{mL}$, the optimal threshold (HRP2:pLDH ratio) for classifying whether samples come from individuals with cleared infection is 8.99 , above which cleared infection due to recent anti-malarial treatment is predicted [area under the curve (AUC): 0.92] (Fig. 6c). Here the sensitivity is $88.9 \%$ and specificity is $89.7 \%$. If HRP2 is $\leq 100 \mathrm{pg} / \mathrm{mL}$, the optimal threshold is 0.49 HRP2:pLDH (AUC: 0.77), here sensitivity $=64.1 \%$ and specificity $=85.7 \%$ (Fig. $6 \mathrm{~d}$ ). Overall, this classification algorithm performed with $77.5 \%$ sensitivity and $88.9 \%$ specificity (AUC: 0.83 ).

Finally, there were 11/614 samples (1.8\%) from eight unique individuals positive by direct skin feeding (DSF) experiments. On average, the HRP2:pLDH ratio was lower $(\mathrm{P}=0.02)$ for individuals with a positive DSF result in reference to microscopy and DSF negative samples (see Additional file 1: Table S1). The small number of individuals positive by DSF, a potential result of transmission-blocking vaccines administered prior to blood draw, limited the extent of our direct skin feeding analysis.

\section{Discussion}

This analysis uses data available from both laboratory and field testing in Bancoumana, Mali, to inform three overarching objectives, to: (1) evaluate performance of the uRDT compared to other diagnostic methods, (2) investigate how relative antigen concentrations can classify infections, and (3) better understand the post-treatment dynamics of pLDH and HRP2.

Comparing the HRP2 threshold at which there was a $50 \%$ probability of detection by uRDT with the same values from two other studies in Uganda and Myanmar [7, 8] resulted in unexpected differences. HRP2 concentrations in Uganda were quantified using a Bi-Plex Human Malaria Array, an earlier version of the Q-Plex ELISA with a lower LOD $(0.1 \mathrm{pg} / \mathrm{mL})$ [7], potentially contributing to observed differences in detection thresholds. Other sources of variation could be (but were not confirmed) lot-to-lot variation in performance of the uRDT, variability in class of HRP2 present at the different locations [20], storage conditions of tests, and/or interpretation of test results. Overall, results indicate a need for further evaluation of the uRDT LOD in the field based on antigenaemia, similar to the large-scale systematic review of co-RDT detection by parasitaemia [21].

In this Malian population, pLDH had a stronger correlation with parasitaemia than HRP2, with the constraint that parasitaemia was quantified by microscopy and not quantitative polymerase chain reaction (qPCR). This is consistent with findings that residual HRP2 lingers after parasite clearance, whereas pLDH has a shorter half-life and is more indicative of active infection [22, 23]. This analysis is important in the context of future development or adoption of pLDH-based assays to address emerging $p f h r p 2 / 3$ deletions $[25,26]$. In this dataset, there were 12 microscopy-positive, HRP2-negative cases, 


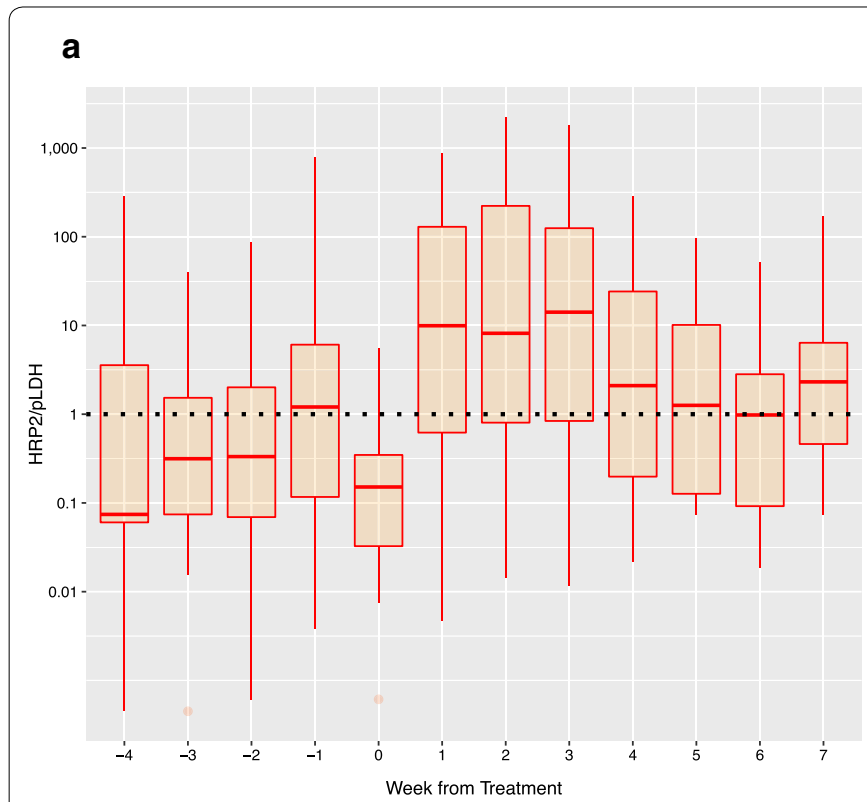

\section{b}

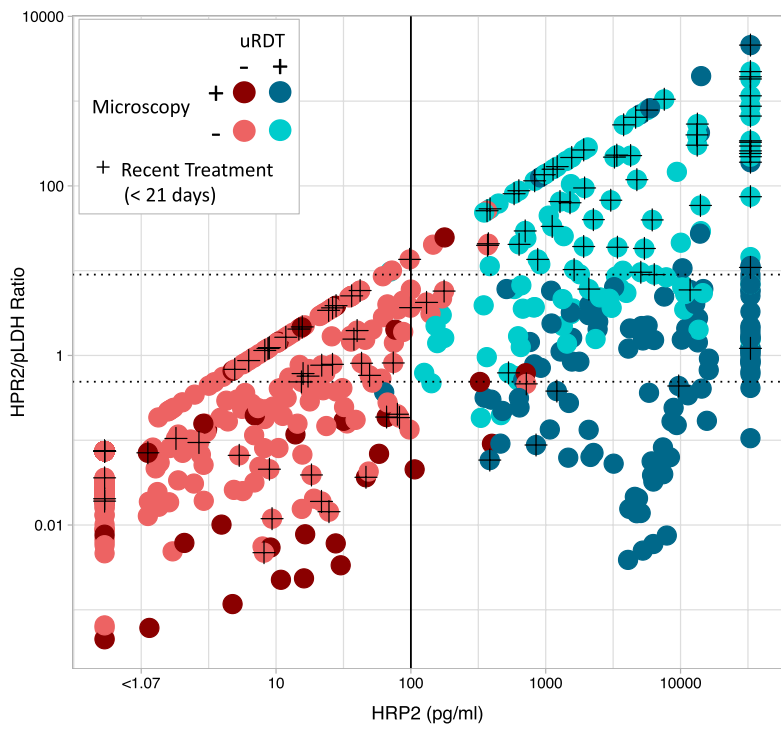

d $\mathrm{HRP} 2<100 \mathrm{pg} / \mathrm{ml}$

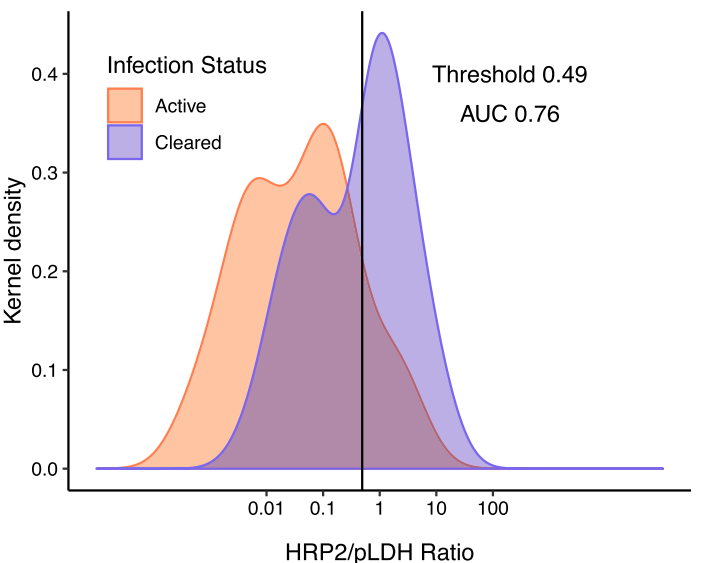

Fig. 6 HRP2:pLDH ratios as tool to predict infection status. a Boxplot of HRP2:pLDH ratios for individuals within 4 weeks pretreatment to 7 weeks post-treatment $(n=311)$. b Scatterplot of the relationship between HRP2:pLDH vs. HRP2. c Density plot of HRP2:pLDH ratios for individuals with active vs. cleared P. falciparum infection and HRP2 $>100 \mathrm{pg} / \mathrm{mL}$. With a cutoff of 8.99 HRP2:pLDH ratio, cleared infection is predicted with sensitivity $=88.9 \%$, specificity $=89.7 \%$, and $A \cup C=0.92$. $\mathbf{d}$ Density plot of HRP2:pLDH ratios for individuals with active vs. cleared infection and HRP2 $\leq 100 \mathrm{pg} / \mathrm{mL}$. With a cutoff of $0.49 \mathrm{HRP} 2 / \mathrm{pLDH}$ ratio, cleared infection is predicted with sensitivity $=64.1 \%$, specificity $=85.7 \%$, and $A \cup C=0.77$. AUC, area under the curve; HRP2, histidine rich protein 2; pLDH, Plasmodium lactate dehydrogenase

4 of which were confirmed to be non-falciparum infections. Of the remaining eight cases, all had low parasite densities $(\leq 20$ parasites $/ \mathrm{mL})$ and only three had a significant $\mathrm{pLDH}$ signal $(>300 \mathrm{pg} / \mathrm{mL}$ ). Further molecular analysis is required to confirm if these are $p f h r p 2 / 3$ deletions $[24,25]$.

Several first-order kinetics models have previously been used to fit HRP2 dynamics [22, 26]. Here biphasic exponential decay models were found to best capture pLDH and HRP2 clearance post-treatment [19]. The nature of biphasic exponential decay (fast, then slow decay) means that previous models may overestimate antigen concentrations initially in the days following treatment [27].

One of the concerns accompanying introduction of the uRDT is that due to HRP2 persistence, ultra-sensitive HRP2-based diagnostics may lead to overtreatment due to individuals with recently cleared infections testing positive and being treated with anti-malarial drugs when there may be another infection or illness causing fever [28]. The ability to use a patient's antigen concentrations 
to predict if they are in a stage of typical antigen decline post-treatment would be beneficial both to avoid unnecessary retreatment with anti-malarial drugs and to better understand levels of active infection in the population. This need to distinguish between previous versus active infection led us to develop a novel algorithm for distinguishing recently cleared infections from active ones based on both HRP2 concentration and HRP2:pLDH ratios. Although pLDH alone can be a reliable indicator of active infection, it can be difficult to classify pLDHpositive infections as active versus recently cleared without detailed drug treatment histories. Therefore, although perhaps not viable as a standard case management tool, our approach could be used for routine monitoring of drug efficacy at sentinel surveillance sites and to improve estimates of prevalence in cross-sectional surveys. Overall sensitivity of the classification algorithm was promising (77.5\%), with predictive power highest for samples with $>100 \mathrm{pg} / \mathrm{mL}$ HRP2. In our analysis, HRP2 and PLDH levels below the LOQ were treated as LOQ/2, although most samples likely cleared $\mathrm{pLDH}$ within the 21-day post-treatment window due its more rapid clearance dynamics. In order for HRP2:pLDH ratios to become a reproducible metric for distinguishing recently cleared $P$. falciparum infections in the future, a standardized protocol for dealing with $\mathrm{pLDH}$ values of $0 \mathrm{pg} / \mathrm{mL}$ (or < LOQ) in HRP2:pLDH calculations will need to be defined.

This analysis was limited to individuals in a hightransmission $P$. falciparum setting and to adults over 18, whereas children under 5 years carry the majority of the global malaria burden [29]. qPCR data was also not collected in this study. Without being able to account for submicroscopic infections, a significant proportion of the infectious reservoir may be being ignored [21]. Further analyses should incorporate data from low-transmission settings and on low-density, submicroscopic infections.

\section{Conclusions}

A deeper understanding of HRP2 and pLDH antigen dynamics in malaria endemic populations will greatly inform the understanding of the performance of RDTs and the utility of RDTs in malaria interventions beyond case management. The data presented here, only considering the relative proportions of two malaria antigens (HRP2 and LDH), suggests that antigen dynamics can be used to differentiate active infections from recently treated infections in P. falciparum cases. Further studies analysing a range of longitudinal datasets that include anti-malarial drug history are required to improve upon and validate this approach.

\section{Supplementary information}

Supplementary information accompanies this paper at https://doi. org/10.1186/s12936-020-03389-0.

\begin{abstract}
Additional file 1: Figure S1. Scatterplot showing correlation between parasite count by microscopy and HRP2 concentration quantified by Q-Plex ELISA $\left(R^{2}=0.29\right)$ for all samples $P$. falciparum positive by microscopy (>0 parasites/ $\mu \mathrm{L}$ ). Abbreviations: ELISA, enzyme-linked immunosorbent assay; HRP2, histidine rich protein 2; pLDH, Plasmodium lactate dehydrogenase. Figure S2. Scatterplot showing correlation between parasite count by microscopy and pLDH concentration quantified by Q-Plex ELISA $\left(R^{2}=0.61\right)$ for all samples $P$. falciparum positive by microscopy $(>0$ parasites $/ \mu \mathrm{L}$ ). Table $\mathbf{S} 1$. Antigen ratios by direct skin feed (DSF) infectivity status. Geometric means for the two antigens of interest, HRP2 and pLDH, along with the geometric mean HRP2:pLDH ratio for study participants that were DSF-positive $(n=11)$ or DSF-negative $(n=603)$, grouped by microscopy result. Individuals positive by DSF had a significantly lower HRP2:pLDH ratio $(p=0.02)$ on average in reference to DSF and microscopy negative individuals. Abbreviations: geo, geometric; HRP2, histidine rich protein 2; pLDH, Plasmodium lactate dehydrogenase; SD, standard deviation.
\end{abstract}

\section{Abbreviations}

AIC: Akaike information criterion; BIC: Bayesian information criterion; Cl: Confidence interval; GM: Geometric mean; HRP2: Histidine rich protein 2; LDH: Lactate dehydrogenase; LMIV: Laboratory of Malaria Immunology and Vaccinology; LOD: Limit of detection; LOQ: Limit of quantification; NIAID: National Institute of Allergy and Infectious Diseases; NIH: National Institutes of Health; pLDH: Plasmodium lactate dehydrogenase; RDT: Rapid diagnostic test; ROC: Receiver operating characteristic; URDT: Ultra-sensitive HRP2-based Alere ${ }^{\mathrm{TM}}$ Malaria Ag P.f RDT; SD: Standard deviation.

\section{Acknowledgements}

The authors would like to thank Ruiyun Li at Imperial College for sharing code, and Christine Waresak and Sophie Knudson for proofing and editing the manuscript.

\section{Authors' contributions}

JCCH, IS, SAH, MHA, MAG, PED contributed to the clinical study design, implementation, and specimen characterization; $R B, A R, A G$, and IKY contributed to sample analysis; ENR, HCS and GJD designed the analyses. ENR drafted the manuscript; HCS, GJD, JCH, SAH, and PED revised the manuscript. All authors read and approved the final manuscript.

\section{Funding}

This work was supported by the Bill \& Melinda Gates Foundation [Grant Number OPP1053616]; the Siemens Foundation-PATH Fellowship [Fellowship Number OPP-00006234 to E.N.R.]; and the National Institutes of Health Intramural Research Program.

\section{Availability of data and materials}

The datasets used and/or analyzed during the current study are available from the corresponding author on reasonable request. $\mathrm{R}$ code for all analyses and figures are publicly available at https://github.com/PATH-Global-Health/Reich ert_Mali_antigens.

\section{Ethics approval and consent to participate}

This Phase I trial was conducted in accordance with Good Clinical Practice guidelines and institutional procedures and guidelines. Each village provided community permission and all participants provided informed consent. The study was approved by the Mali ethics review board (Faculté de Médecine de Pharmacie et d'OdontoStomatologie, Bamako), U.S. National Institute of Allergy and Infectious Diseases (NIAID, National Institutes of Health [NIH], Bethesda, MD) institutional review board, and Mali national regulatory authority, and was conducted under FDA IND 16251. 


\section{Consent for publication \\ Not applicable.}

\section{Competing interests}

The authors declare that they have no competing interests.

\begin{abstract}
Author details
${ }^{1}$ Diagnostics Program, PATH, Seattle, WA, USA. ${ }^{2}$ Laboratory of Malaria Immunology and Vaccinology, National Institute of Allergy and Infectious Diseases, National Institutes of Health, Rockville, MD, USA. ${ }^{3}$ Malaria Research and Training Center, Mali-National Institute of Allergy and Infectious Diseases International Center for Excellence in Research, University of Science, Techniques and Technologies of Bamako, Bamako, Mali.
\end{abstract}

Received: 2 June 2020 Accepted: 25 August 2020 Published online: 03 September 2020

\section{References}

1. Chiodini PL. Malaria diagnostics: now and the future. Parasitology. 2014;141:1873-9.

2. Mukkala AN, Kwan J, Lau R, Harris D, Kain D, Boggild AK. An update on malaria rapid diagnostic tests. Curr Infect Dis Rep. 2018:20:49.

3. Dalmat R, Naughton B, Kwan-Gett TS, Slyker J, Stuckey EM. Use cases for genetic epidemiology in malaria elimination. Malar J. 2019;18:163.

4. Roth JM, Korevaar DA, Leeflang MMG, Mens PF. Molecular malaria diagnostics: a systematic review and meta-analysis. Crit Rev Clin Lab Sci. 2016;53:87-105.

5. WHO. Malaria rapid diagnostic test performance. Results of $\mathrm{WHO}$ product testing of malaria RDTs: round 8 (2016-2018). Geneva: World Health Organization. https://www.who.int/malaria/publications/atoz/97892 41514965/en/. Accessed 21 July 2020

6. Wu L, van den Hoogen LL, Slater H, Walker PG, Ghani AC, Drakeley CJ, et al. Comparison of diagnostics for the detection of asymptomatic Plasmodium falciparum infections to inform control and elimination strategies. Nature. 2015;528:S86-93.

7. Das S, Jang IK, Barney B, Peck R, Rek JC, Arinaitwe E, et al. Performance of a high-sensitivity rapid diagnostic test for Plasmodium falciparum malaria in asymptomatic individuals from Uganda and Myanmar and naive human challenge infections. Am J Trop Med Hyg. 2017;97:1540-50.

8. Landier J, Haohankhunnatham W, Das S, Konghahong K, Christensen P, Raksuansak J, et al. Operational performance of a Plasmodium falciparum ultrasensitive rapid diagnostic test for detection of asymptomatic infections in Eastern Myanmar. J Clin Microbiol. 2018;56:e00565-18.

9. Jang IK, Tyler A, Lyman C, Kahn M, Klnoky M, Rek JC, et al. Simultaneous quantification of Plasmodium antigens and host factor $\mathrm{C}$-reactive protein in asymptomatic individuals with confirmed malaria by use of a novel multiplex immunoassay. J Clin Microbiol. 2019;57:e00948-18.

10. Plucinski MM, Dimbu PR, Fortes F, Murphy SC, Smith NT, Cruz KR, et al. Malaria parasite density in individuals with different rapid diagnostic test results and concentrations of HRP2 antigen. Am J Trop Med Hyg. 2019:100:1202-3.

11. Plucinski MM, Rogier E, Dimbu PR, Fortes F, Halsey ES, Aidoo M. Estimating the added utility of highly sensitive histidine-rich protein 2 detection in outpatient clinics in sub-Saharan Africa. Am J Trop Med Hyg. 2017:97:1159-62.

12. Plucinski MM, Rogier E, Dimbu PR, Fortes F, Halsey ES, Aidoo M, et al. Performance of antigen concentration thresholds for attributing fever to malaria among outpatients in Angola. J Clin Microbiol. 2019;57:e01901-18.

13. Plucinski M, Dimbu R, Candrinho B, Colborn J, Badia A, Ndiaye D, et al. Malaria surveys using rapid diagnostic tests and validation of results using post hoc quantification of Plasmodium falciparum histidine-rich protein 2. Malar J. 2017;16:451

14. Rogier E, Plucinski M, Lucchi N, Mace K, Chang M, Lemoine JF, et al. Beadbased immunoassay allows sub-picogram detection of histidine-rich protein 2 from Plasmodium falciparum and estimates reliability of malaria rapid diagnostic tests. PLoS ONE. 2017;12:e0172139.
15. Das S, Peck RB, Barney R, Jang IK, Kahn M, Zhu M, et al. Performance of an ultra-sensitive Plasmodium falciparum HRP2-based rapid diagnostic test with recombinant HRP2, culture parasites, and archived whole blood samples. Malar J. 2018;17:118.

16. CDC -DPDx-Diagnostic procedures_-blood specimens. Published January 8, 2019. https://www.cdc.gov/dpdx/diagnosticprocedures/blood /microexam.html. Accessed 9 Sept 2019

17. Nyunt MH, Kyaw MP, Win KK, Myint KM, Nyunt KM. Field evaluation of HRP2 and pan pLDH-based immunochromatographic assay in therapeutic monitoring of uncomplicated falciparum malaria in Myanmar. Malar J. 2013;12:123.

18. R Core Team. R: a language and environment for statistical computing. R Foundation for Statistical Computing, Vienna, Austria; 2018. https:// www.R-project.org/.

19. Plucinski MM, McElroy PD, Dimbu PR, Fortes F, Nace D, Halsey ES, et al. Clearance dynamics of lactate dehydrogenase and aldolase following antimalarial treatment for Plasmodium falciparum infection. Parasit Vectors. 2019:12:293.

20. Baker J, Ho M-F, Pelecanos A, Gatton M, Chen N, Abdullah S, et al. Global sequence variation in the histidine-rich proteins 2 and 3 of Plasmodium falciparum: implications for the performance of malaria rapid diagnostic tests. Malar J. 2010:9:129.

21. Slater HC, Ross A, Felger I, Hofmann NE, Robinson L, Cook J, et al. The temporal dynamics and infectiousness of subpatent Plasmodium falciparum infections in relation to parasite density. Nat Commun. 2019;10:1433.

22. Marquart L, Butterworth A, McCarthy JS, Gatton ML. Modelling the dynamics of Plasmodium falciparum histidine-rich protein 2 in human malaria to better understand malaria rapid diagnostic test performance. Malar J. 2012;11:74

23. Mayxay M, Pukrittayakamee S, Chotivanich K, Looareesuwan S, White NJ. Persistence of Plasmodium falciparum HRP-2 in successfully treated acute falciparum malaria. Trans R Soc Trop Med Hyg. 2001;95:179-82.

24. Cheng Q, Gatton ML, Barnwell J, Chiodini P, McCarthy J, Bell D, et al. Plasmodium falciparum parasites lacking histidine-rich protein 2 and 3: a review and recommendations for accurate reporting. Malar J. 2014;13:283.

25. Gatton ML, Dunn J, Chaudhry A, Ciketic S, Cunningham J, Cheng Q. Implications of parasites lacking Plasmodium falciparum histidine-rich protein 2 on malaria morbidity and control when rapid diagnostic tests are used for diagnosis. J Infect Dis. 2017;215:1156-66.

26. Plucinski MM, Dimbu PR, Fortes F, Abdulla S, Ahmed S, Gutman J, et al. Posttreatment HRP2 clearance in patients with uncomplicated Plasmodium falciparum malaria. J Infect Dis. 2018;217:685-92.

27. Brouwer AF, Eisenberg MC, Remais JV, Collender PA, Meza R, Eisenberg JNS. Modeling biphasic environmental decay of pathogens and implications for risk analysis. Environ Sci Technol. 2017;51:2186-96.

28. Dalrymple U, Cameron E, Arambepola R, Battle KE, Chestnutt EG, Keddie $\mathrm{SH}$, et al. The contribution of non-malarial febrile illness co-infections to Plasmodium falciparum case counts in health facilities in sub-Saharan Africa. Malar J. 2019;18:195.

29. WHO. This year's World malaria report at a glance. Geneva: World Health Organization; 2018. http://www.who.int/malaria/media/world-malar ia-report-2018/en/. Accessed 7 Oct 2019.

30. Heutmekers M, Gillet P, Maltha J, Scheirlinck A, Cnops L, Bottieau E, et al. Evaluation of the rapid diagnostic test CareStart pLDH Malaria (Pf-pLDH/ pan-pLDH) for the diagnosis of malaria in a reference setting. Malar J. 2012;11:204.

31. Jimenez A, Rees-Channer RR, Perera R, Gamboa D, Chiodini PL, Gonzalez IJ, et al. Analytical sensitivity of current best-in-class malaria rapid diagnostic tests. Malar J. 2017:16:128.

\section{Publisher's Note}

Springer Nature remains neutral with regard to jurisdictional claims in published maps and institutional affiliations. 